\title{
Collapse arresting in an inhomogeneous two-dimensional nonlinear Schrodinger model
}

Schjødt-Eriksen, Jens; Gaididei, Yuri Borisovich; Christiansen, Peter Leth

Published in:

Physical Review E. Statistical, Nonlinear, and Soft Matter Physics

Link to article, DOI:

10.1103/PhysRevE.64.066614

Publication date:

2001

Document Version

Publisher's PDF, also known as Version of record

Link back to DTU Orbit

Citation (APA):

Schjødt-Eriksen, J., Gaididei, Y. B., \& Christiansen, P. L. (2001). Collapse arresting in an inhomogeneous twodimensional nonlinear Schrodinger model. Physical Review E. Statistical, Nonlinear, and Soft Matter Physics, 64(6), 066614. https://doi.org/10.1103/PhysRevE.64.066614

\section{General rights}

Copyright and moral rights for the publications made accessible in the public portal are retained by the authors and/or other copyright owners and it is a condition of accessing publications that users recognise and abide by the legal requirements associated with these rights.

- Users may download and print one copy of any publication from the public portal for the purpose of private study or research.

- You may not further distribute the material or use it for any profit-making activity or commercial gain

- You may freely distribute the URL identifying the publication in the public portal 


\title{
Collapse arresting in an inhomogeneous two-dimensional nonlinear Schrödinger model
}

\author{
Jens Schjødt-Eriksen, ${ }^{*}$ Yu. B. Gaididei ${ }^{\dagger}$ and P. L. Christiansen ${ }^{\sharp}$ \\ Informatics and Mathematical Modelling, Technical University of Denmark, DK-2800 Lyngby, Denmark \\ (Received 14 November 2000; revised manuscript received 12 February 2001; published 26 November 2001) \\ Collapse of $(2+1)$-dimensional beams in the inhomogeneous two-dimensional cubic nonlinear Schrödinger \\ equation is analyzed numerically and analytically. It is shown that in the vicinity of a narrow attractive \\ inhomogeneity, the collapse of beams that in a homogeneous medium would collapse may be arrested under \\ certain circumstances.
}

DOI: 10.1103/PhysRevE.64.066614

PACS number(s): 42.65.Jx, 05.45.-a

\section{INTRODUCTION}

In the framework of the nonlinear Schrödinger equation (NLSE) or alternatively denoted the Gross-Pitaevskii equation in Bose-Einstein condensation (BEC), the interaction of excitations with potentials has attracted significant interest during recent years. Due to the universality of the NLSE in the context of weakly nonlinear dispersive media, these fundamental investigations apply to such diverse phenonema as light beams trapped in waveguides $[1,2]$, molecular excitations in the vicinity of inhomogeneities [3], and BoseEinstein condensation $[4,5]$. In this paper, the investigations are based on the following variant of the NLSE:

$$
i \partial_{z} \psi+\nabla_{\perp}^{2} \psi+|\psi|^{2} \psi+V(\vec{r}) \psi=0
$$

where $\psi \equiv \psi(\vec{r}, z)$ is the complex amplitude of the quasimonochromatic wave train (the condensate wave function in $\mathrm{BEC}), \nabla_{\perp}^{2}=\partial_{x}^{2}+\partial_{y}^{2}$ is the two-dimensional Laplace operator accounting for diffraction, $\mathrm{z}$ is the propagation variable (the time variable in $\mathrm{BEC})$ and $\vec{r}=(x, y)$ is the spatial coordinate. The nonlinear term in Eq. (1), $\left.|\psi|\right|^{2} \psi$, characterizes the nonlinear properties of the system; light intensity-dependent refractive index in optics, the interaction between Boseparticles in BEC. Finally, the potential, $V(\vec{r})$, e.g., represents a space-dependent linear refractive index of an optical medium, a confining applied field in BEC, or a material inhomogeneity in the theory of trapped molecular excitations. In the present paper, we restrict ourselves to consider a real attractive potential that decays monotonically as a function of the distance from the center $\vec{r}=\overrightarrow{0}$ of the $\vec{r}$ plane. In physical systems where an excitation is located in the vicinity of a smooth bell-shaped potential whose width is much larger than the width of the excitation, one may approximate the potential by a parabola. Many important results relating to the dynamics of excitations in the vicinity of parabolic potentials have been obtained. For a short summary and references to these works, see our previous paper [6]. However, the parabolic approximation breaks down when the widths of

\footnotetext{
*Email address: jse@imm.dtu.dk

${ }^{\dagger}$ Permanent address: Bogolyubov Insitute for Theoretical Physics, 252143 Kiev, Ukraine; email address: yg@imm.dtu.dk

‡Email address: plc@imm.dtu.dk
}

the potential and the excitation are of comparable size. In [6] we used the one-dimensional (1D) quintic NLSE to model the propagation of 1D "beams" in the presence of a narrow attractive potential. Similar to the cubic NLSE, the quintic NLS model has a threshold for collapse. We launched a supercritical beam into the model and varied the initial distance $R_{0}$ between beam and potential. For large values of $R_{0}$, the interaction of beam and potential was found to be neglegible and the beam collapsed as expected. Collapse was also observed for small initial distances; here, the effect of the potential was to shorten the propagation distance needed for a collapse to occur compared to the homogeneous case $[V(\vec{r})$ $=\overrightarrow{0}]$. However, it was observed that the collapse could be arrested if $R_{0}$ lay in a certain interval. In this case, the beam acceleration induced by the potential was strong enough to separate the beam into radiation and a noncollapsing core oscillating in the potential.

In this paper, we extend the previous $1 \mathrm{D}$ investigations to the physically more relevant cubic NLSE and obtain qualitatively similar results for rectilinear motion of the $2 \mathrm{D}$ beam in the transverse plane. However, we also present new manifestations of the collapse arresting due to the two degrees of freedom in the transverse plane of the cubic NLSE. In particular, we demonstrate how the collapse of a supercritical beam may also be arrested for circular motion of the beam center around the potential.

The paper is organized as follows. In Sec. II, we introduce the model and describe its basic properties in the homogeneous case before discussing the numerical results obtained when a potential is included in the model. In Sec. III, we address the problem analytically. Using a certain coordinate transformation enables us to calculate energy radiation from the beam using methods developed to characterize the tunneling of probability density in linear quantum-mechanical systems. Finally, Sec. IV summarizes our results.

\section{MODEL AND NUMERICAL RESULTS}

To model the propagation of a $(2+1)$-dimensional excitation, $\psi(x, y, z)$, which we in the following shall refer to as a beam, we use the NLSE as given by Eq. (1) where $\psi$ $\equiv \psi(\vec{r}, z), \vec{r}=(x, y)$ is the transverse coordinate, $\nabla_{\perp}^{2}=\partial_{x}^{2}$ $+\partial_{y}^{2}$ is the Laplacian governing diffraction in the transverse plane, and $z$ measures propagation length. In the homoge- 
neous case $[V(\vec{r})=0]$, Eq. (1) has stationary solutions of the form

$$
\psi(x, y, z)=\Psi(x, y, \Lambda) e^{i \Lambda z},
$$

where the real shape function $\Psi(x, y, \Lambda)$ satisfies the equation

$$
\begin{aligned}
& \frac{\partial^{2} \Psi(x, y, \Lambda)}{\partial x^{2}}+\frac{\partial^{2} \Psi(x, y, \Lambda)}{\partial y^{2}}+\Psi^{3}(x, y, \Lambda)-\Lambda \Psi(x, y, \Lambda) \\
& \quad=0 .
\end{aligned}
$$

From Eq. (3), the well-known family of self-similar stationary solutions [7]

$$
\Psi(x, y, \Lambda)=\sqrt{\frac{\Lambda}{\Lambda_{0}}} \Psi_{0}\left(\sqrt{\frac{\Lambda}{\Lambda_{0}}} x, \sqrt{\frac{\Lambda}{\Lambda_{0}}} y, \Lambda_{0}\right),
$$

appears, once one solution, $\Psi_{0}$ with frequency $\Lambda_{0}$, has been found. It then follows that the mass $N$ of the stationary solutions

$$
N(\Lambda)=\int_{-\infty}^{\infty} \int_{-\infty}^{\infty}|\Psi(x, y, \Lambda)|^{2} d x d y
$$

is independent of $\Lambda$ and has a value, which by means of numerical methods, may be evaluated to $N(\Lambda)=N_{c}=11.69$. According to the Vakhitov-Kolokolov criterion [8], the $\Lambda$-independent norm implies marginal stability of the stationary solutions; i.e., if a stationary solution is perturbed such that $N>N_{c}$, a singularity in $|\psi|$ appears after finite propagation length. On the other hand, $\psi$ cannot remain localized if $N<N_{c}$ and ultimately disperses completely.

We restrict ourselves to initial conditions of the form

$$
\psi(x, y, z=0)=A \exp \left(-\frac{\left|\vec{r}-\vec{R}_{0}\right|^{2}}{2 w^{2}}-i \vec{b} \cdot\left(\vec{r}-\vec{R}_{0}\right)\right),
$$

where $\vec{R}_{0}$ is the center of the beam at $z=0$ and $\vec{b}$ controls the initial "velocity" of the center. When the mass $N=\pi A^{2} w^{2}$ approximates the ground-state mass $N_{c}=11.69$, the Gaussian initial conditions approximate a member of the self-similar solution family in Eq. (4) fairly well. This is indeed the case for the values $A=2, w=0.975(N=11.95)$, which are used in all numerical calculations. In Appendix A, we use a Crank-Nicholson finite-difference scheme with an adaptive integration step on a nonuniform grid to determine the propagation distance $z_{0}$ needed for a blow up to occur in the homogeneous case $[V(\vec{r})=0]$. For the values of $A$ and $w$ listed above, $z_{0}$ is found to be 5.45 .

For the potential $V(\vec{r})$ we use a smoothed version of the circular step potential

$$
V(\vec{r})=\epsilon \theta(a-|\vec{r}|)
$$

where $\theta$ is the Heaviside step function and $\epsilon$ and $2 a$ are the height and diameter of the potential, respectively. To monitor how the center of the beam evolves from its initial position $\vec{R}_{0}$ we use the centroid

$$
\vec{R}(z)=\frac{1}{N} \int_{-\infty}^{\infty} \int_{-\infty}^{\infty} \vec{r}|\psi(x, y, z)|^{2} d x d y .
$$

Using Eqs. (1) and (8), the well-known expression for the second derivative of the centroid appears

$$
\vec{R}_{z z}(z)=\frac{2}{N} \int_{-\infty}^{\infty} \int_{-\infty}^{\infty} \nabla_{\perp} V(x, y)|\psi(x, y, z)|^{2} d x d y .
$$

Insertion of Eq. (6) into Eq. (9) yields

$$
\begin{aligned}
\vec{R}_{z z}(z=0)= & \frac{4 A^{2}}{N w^{2}} \int_{-\infty}^{\infty} \int_{-\infty}^{\infty} \mathrm{dx} \mathrm{dy} \\
& \times\left\{\left(\vec{r}_{\|}-\vec{R}_{0}\right) V(x, y) \exp \left(-\frac{\left|\vec{r}-\vec{R}_{0}\right|^{2}}{w^{2}}\right)\right\},
\end{aligned}
$$

where $\vec{r}$ has been decomposed into the two components $\vec{r}_{\|}$ and $\vec{r}_{\perp}$, which are parallel and perpendicular to $\vec{R}_{0}$, respectively. Noting that the potential $V(x, y)$ holds the largest values in the half plane where $\vec{r}_{\|}$points in the opposite direction of $\vec{R}_{0}, \vec{R}_{z z}(z=0)$ is seen to point towards the center of the potential, $\vec{r}=0$, for the Gaussian initial conditions employed here. As in our previous paper [6], we aim to illustrate how the beam may be separated into radiation and a noncollapsing core, due to the attraction towards the interior of the potential. To meet this end, we divide the numerical calculations into the following two groups that give a broad representation of the possible scenarios of beam/potential interaction, without exhausting all types of initial configurations implied by Eq. (6):

(1) Rectilinear motion: the beam is initially placed at nonzero distance $\left(\left|\vec{R}_{0}\right|>0\right)$ from the center of the potential $\vec{r}$ $=\overrightarrow{0}$ with zero velocity $\left[\vec{R}_{z}(z=0)=\overrightarrow{0}\right]$ in the transverse plane.

(2) Orbital motion: the beam is initially placed at nonzero distance $\left(\left|\vec{R}_{0}\right|>0\right)$ from the center of the potential $\vec{r}=\overrightarrow{0}$ with the velocity vector $\vec{R}_{z}(z=0)$ being perpendicular to $\vec{R}_{0}$.

Initial conditions belonging to the first group are "pseudo-2D" and resemble the quintic case [6], whereas the second group fully exploits the two degress of freedom in the transverse plane. Figure 1 shows the result of a numerical calculation belonging to the first category. A beam characterized by $A=2, w=0.975, \vec{b}=\overrightarrow{0}$, and $\left|\vec{R}_{0}\right|=0.75$ is launched into a potential with $\epsilon$ and $a$ given by 1 and 1.25, respectively. In Fig. 1(a), the evolution of the $y=0$ cross section of $|\psi|,|\psi(x, y=0, z)|$, is plotted. We observe how the beam initially focuses as it is accelerated towards the center of the 


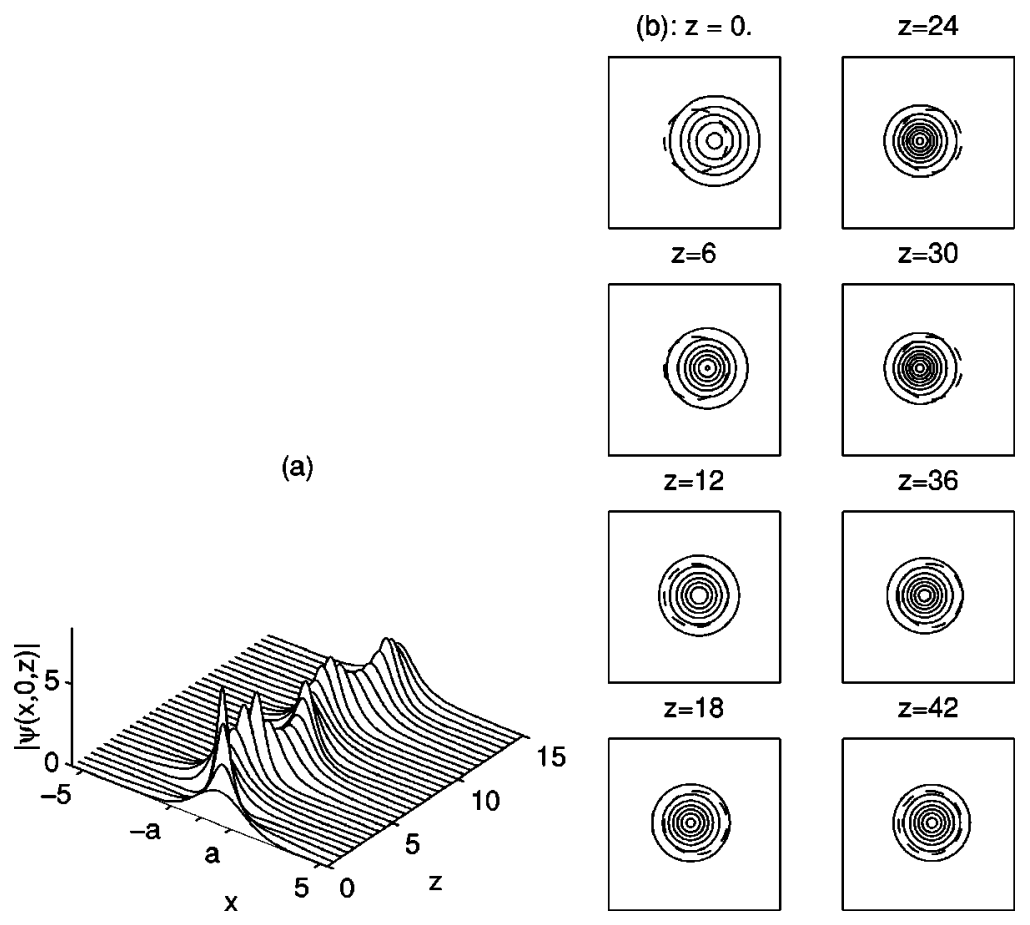

FIG. 1. The following initial conditions for $\psi$ are used: $\psi(x, y, z=0)=A \exp \left(-\left|\vec{r}-\vec{R}_{0}\right|^{2} / 2 w^{2}\right.$ $\left.-i \vec{b} \cdot\left(\vec{r}-\vec{R}_{0}\right)\right)$, where $A=2, w=0.975, \vec{b}=\overrightarrow{0}$ and $\left|\vec{R}_{0}\right|=0.75$. The half diameter $a$ and the height $\epsilon$ of the potential are given by 1.25 and 1 , respectively. In (a), the propagation of the $y=0$ cross section of $|\psi|,|\psi(x, y=0, z)|$, is plotted for dimensionless units of $|\psi|, z$, and $x$. In (b), eight contour plots depict the propagation of $\psi$. The following contour levels are used: $|\psi|$ $=0.4,-0.8,1.1,1.5,1.9,2.3,2.7,3.0$. The potential is indicated by a dashed circle and . potential $\vec{r}=\overrightarrow{0}$. However, the beam amplitude ceases to increase at some point and instead undergoes moderate oscillations. In Fig. 1(b), the propagation of $\psi$ is shown in contour plots for different values of $z$. Eight different contour levels are used to render the beam, and a dashed circle indicates the potential. Few visible contour levels thus correspond to low-beam amplitudes whereas more levels are rendered for higher amplitudes. From the contour plots, it is evident how the beam even at $z=42$, almost eight times $z_{0}$, shows no signs of approaching a collapse. Moreover, we observe how the beam profile remains almost circular through the oscillations in amplitude and width. In order to gain insight into how the beam propagation changes as function of the initial position of the centroid $\vec{R}_{0}$, we fix $A, w, \vec{b}$, $\epsilon$, and $a$ at the values listed above and vary $\vec{R}_{0}$ (Figs. 2, 3, 4). For the numerical calculation shown in Fig. 2, where $\left|\vec{R}_{0}\right|$ $=0.5$, we observe how the beam amplitude increases from 2 to approximately 15 in roughly 1.5 propagation units. Within the validity of the employed split-step integration scheme, this is indicative of a blow up, and no stabilized propagation is thus observed for $\left|\vec{R}_{0}\right|=0.5$. In Fig. 3 , the beam is initially positioned at $\vec{R}_{0}=(1.5,0)$ and similar to Fig. 1 oscillations in

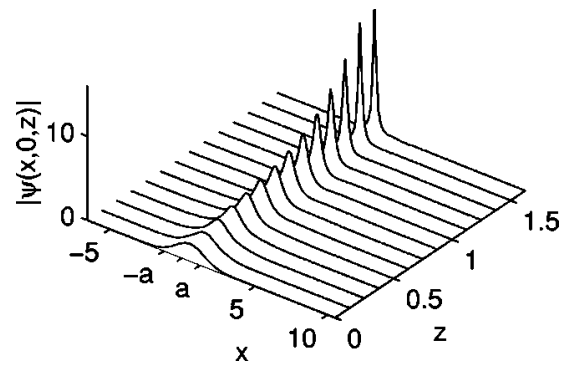

FIG. 2. Same as Fig. 1(a) with $\left|\vec{R}_{0}\right|=0.5$. the beam amplitude past $z_{0}$ are observed. Finally, Fig. 4 depicts the result of a calculation with $\left|\vec{R}_{0}\right|=5.0$. In this case, the overlap between potential and beam is very little and accordingly, the beam undergoes critical self-focusing as in the homogeneous case.

When performing numerical calculations with orbital motion, we borrow concepts from celestial mechanics. For a planet moving in a circular orbit, the magnitude of the acceleration $a_{o r b}$ is related to the radius of the orbit $R_{o r b}$ and the magnitude of the velocity $v_{\text {orb }}$ according to

$$
a_{o r b}=\frac{v_{o r b}^{2}}{R_{o r b}} .
$$

In the present context of an orbiting beam, Eq. (11) translates into

$$
\left|\vec{R}_{z z}(z=0)\right|=\frac{\left|\vec{R}_{z}(z=0)\right|^{2}}{\left|\vec{R}_{0}\right|} .
$$

For fixed values of $A, w, \epsilon$, and $a$, Eq. (9) gives $\left|\vec{R}_{z z}(z=0)\right|$ as a function of $\left|\vec{R}_{0}\right|$. As $\vec{R}_{z}(z=0)=-2 \vec{b}$ for the initial condtions in Eq. (6), Eq. (12) then determines the

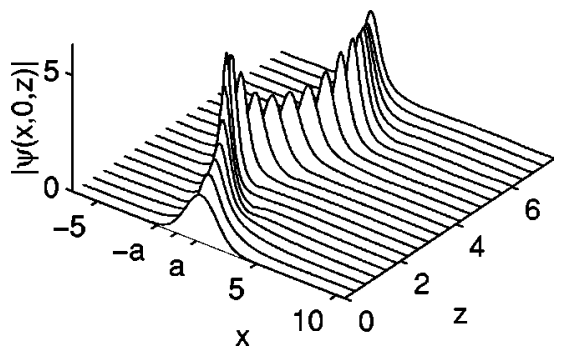

FIG. 3. Same as Fig. 1(a) with $\left|\vec{R}_{0}\right|=1.25$. 


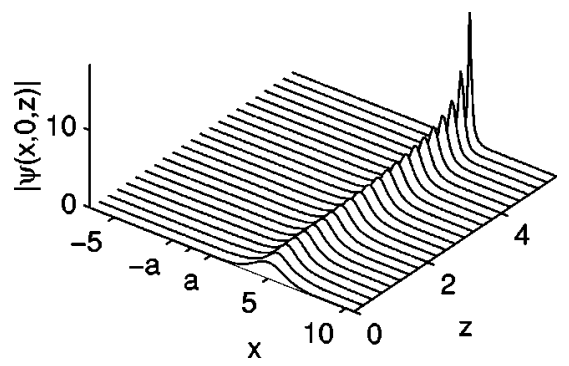

FIG. 4. Same as Fig. 1(a) with $\left|\vec{R}_{0}\right|=5.0$.

$\vec{b}$ vector required for a circular orbit as a function of the other initial parameters. In order to investigate whether sustained circular beam motion is possible, we perform a series of numerical calculations for varying initial parameters and different potential characteristics. In Fig. 5, eight contour plots depict $|\psi|$ as a function of $z$ for initial parameters given by $A=2, w=0.975,\left|\vec{R}_{0}\right|=1.5$, and $\vec{b}=(0,-0.562)$. These initial parameters obey the relation Eq. (12) above, and we observe how the beam orbits one round from $z=0$ to $z=6$ with a fairly constant shape-contour levels appear roughly as circles. However, after $z=6$, the beam profile starts getting somewhat distorted and the beam center moves from the boundary of the potential towards the interior. As in Figs. 1 and 3, we observe how the beam propagates without approaching a collapse. However, in contrast to the scenario of rectilinear motion, the stabilization is not caused by a rapid change in the magnitude of the centroid. On the other hand,

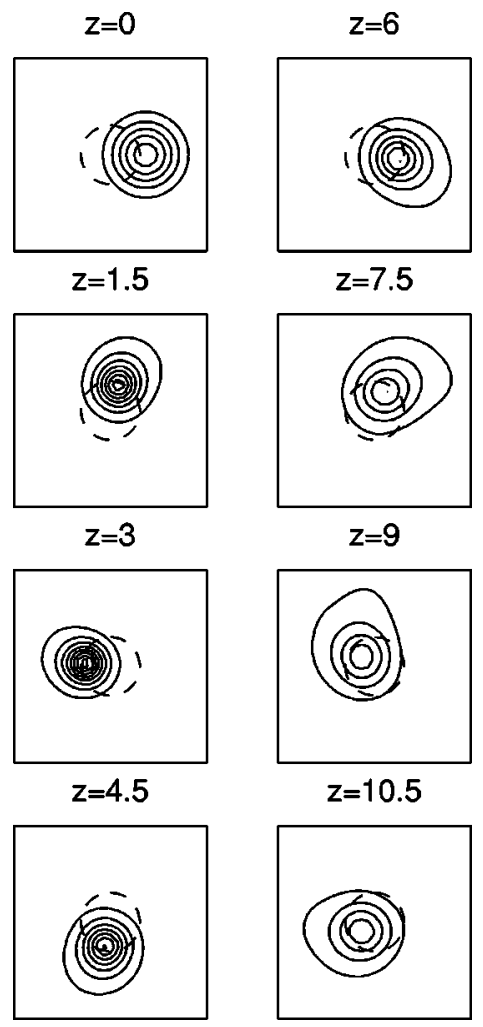

FIG. 5. Same as Fig. 1(b) with $\vec{b}=(0,-0.562),\left|\vec{R}_{0}\right|=1.5$, and contour levels given by $|\psi|=0.3,0.7,1.0,1.7,2.1,2.4,2.8$. the centroid acceleration is in the present case almost solely due to a change in the direction of the centroid vector $\vec{R}_{0}$. In Fig. 6, we change the initial conditions slightly in order to investigate how the beam propagation depends on $\vec{b}$. The relation Eq. (12) is not used in this case, and instead, the magnitude of the $\vec{b}$ vector is increased from 0.562 to 0.7 , while all other parameters are as listed above in the context of Fig. 5. As is visible from the first four subplots in Fig. 6, the beam profile is first distorted severely, followed by a separation process where the beam is divided into a core part trapped at the center of the potential and radiation escaping from the potential. This is evident at $z=9$ and $z=10.5$ where the orbitting motion has stopped and the beam and potential centers approximately coincide. A distorted beam profile is also observed if we again use Eq. (12) to calculate $\vec{b}$ from the other initial parameters whose values are now given by $A$ $=2, w=0.975,\left|\vec{R}_{0}\right|=2.0$. The increase in the magnitude of the initial centroid vector leads to unstable orbitting motion with a clear separation of the beam into a core and radiation (not shown). Finally, a calculation is performed with the same initial parameters as in Fig. (5) apart from a wider potential. According to Eq. (9), Eq. (12) holds for $a=1.25$ as well as $a=2.1$ if $A=2, w=0.975, \vec{b}=(0,-0.562),\left|\vec{R}_{0}\right|$ $=1.5$, and $\epsilon=1$. Results are shown in Fig. 7 where we note how orbiting motion on the inside of the wide potential is significantly more stable compared to propagation on the outside of the narrow potential shown in Fig. 1. The calculation is continued until $z=45$ (almost 4.5 times the propa-

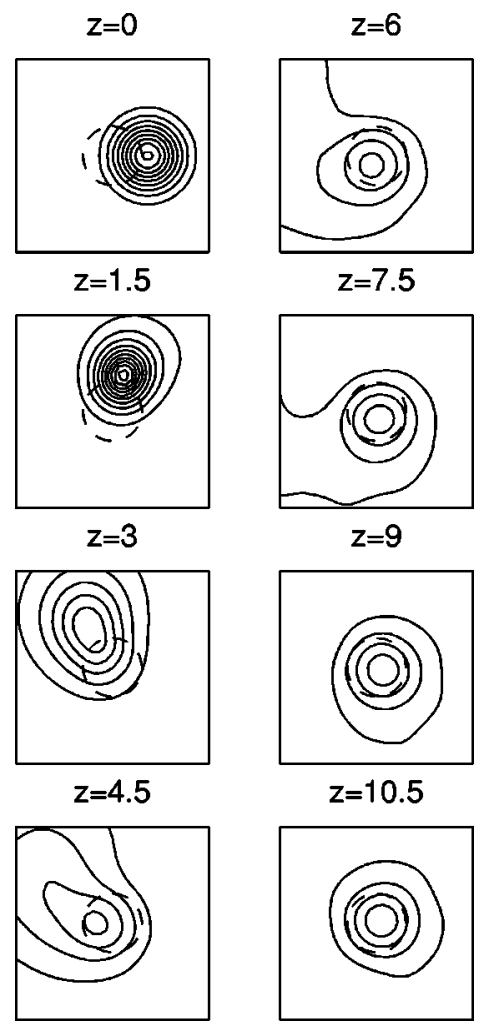

FIG. 6. Same as Fig. 1(b) with $\vec{b}=(0,-0.7),\left|\vec{R}_{0}\right|=1.5$, and contour levels given by $|\psi|=0.2,0.4,0.6,0.9,1.1,1.3,1.5$, $1.7,1.9,2.2$ 


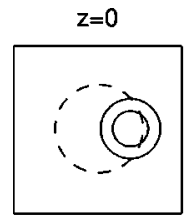

$z=3$

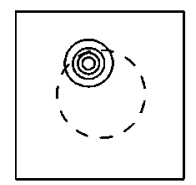

$\mathrm{z}=6$

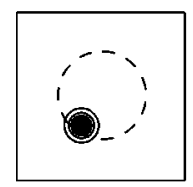

$z=9$

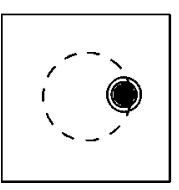

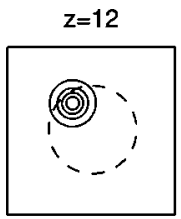

$z=15$

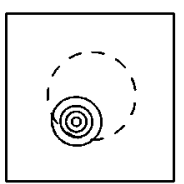

$z=18$

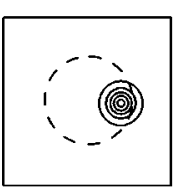

$z=21$

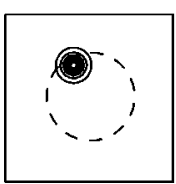

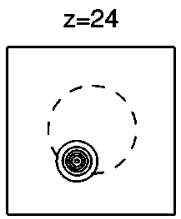

$z=27$

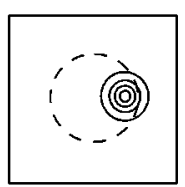

$\mathrm{z}=30$

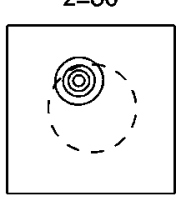

$z=33$

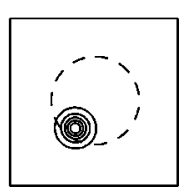

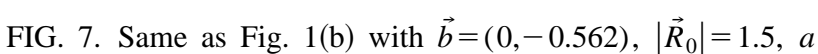
$=2.1$, and contour levels given by $|\psi|=0.7,1.4,2.1,2.7$, 3.4, 4.1, 4.8, 5.5.

gation length from Fig. 5) and not even at this point does the beam show signs of significant distortion. The beam profile visible in the contour plots does in fact appear to be circlelike through all stages of propagation. Moreover, the orbiting motion is accompanied by oscillations in the amplitude and width of the beam that decrease in strength through the propagation.

In conclusion, we have for a variety of initial conditions demonstrated how an initially super-critical beam may be separated into radiation and a noncollapsing core. We rely on physical arguments in concluding that the core mass must be below the critical value $N_{c}=11.69$ required for self focusing; from various theoretical studies (see, e.g., [9]), attractive potentials, as the one in Eq. (7), are known to support stationary solutions having subcritical masses. As the noncollapsing beams propagate with moderate oscillations in the width, amplitude, and centroid for large values of $z$ [see, e.g., Fig. (1)], we expect the core to approximate one of the stationary solutions reasonably well, and thus to have a subcritical mass. Trying to calculate the core mass exactly would be ambiguous, since the boundary between core and radiation is not well defined. Indeed, positioning the boundary too far from the center of the potential would yield a super-critical value of the core mass.

In the following Sec. III, where the numerically observed phenomena are subject to analytical treatment, the beam is decomposed into radiation and a self-similar core with varying mass. In doing so we obtain a qualitative explanation of the collapse arresting.

\section{ANALYTICAL RESULTS}

In order to give an analytical description of the process, we generalize the approach that was used in our previous paper where we investigated collapse arresting in a onedimensional inhomogeneous quintic nonlinear Schrödinger model [6]. First, we introduce the transformation to the noninertial frame of reference in which the centroid of the beam is at rest. Thus,

$$
\psi(\vec{r}, z)=\phi(\vec{\rho}, z) \exp \left(i \vec{k}(z) \vec{r}+i \int_{0}^{z} \vec{k}^{2}\left(z^{\prime}\right) d z^{\prime}\right),
$$

where $\vec{\rho}=\vec{r}-\vec{R}(z)$ is the transversal coordinate in the frame of reference, and $\vec{k}(z)=(1 / 2) \dot{\vec{R}}$ is the momentum canonically conjugated to the centroid coordinate (Overdot denotes the derivative $d / d z$ ). In the frame of reference Eq. (1) takes the form

$$
i \phi_{z}+\nabla_{\rho}^{2} \phi+|\phi|^{2} \phi+V[\vec{\rho}+\vec{R}(z)] \phi-\frac{1}{2} \ddot{\vec{R}} \vec{\rho} \phi=0 \text {. }
$$

The centroid coordinate $\vec{R}(z)$ satisfies the equation

$$
\frac{1}{2} \ddot{\vec{R}}=\frac{1}{N} \int_{-\infty}^{\infty}|\psi(\vec{r}, z)|^{2} \nabla_{r} V(\vec{r}) d \vec{r}
$$

The fourth term in the left-hand side of Eq. (14) describes the influence of the linear potential in the frame of reference, while the fifth term represents the inertial force work. It is worth noticing that due to Eqs. (14) and (15), the function $\phi(\vec{r}, z)$ should satisfy the following compatibility condition:

$$
\int_{-\infty}^{\infty} \vec{r}|\phi(\vec{r}, z)|^{2} d x=0
$$

Using the lens transformation, used in the homogeneous case in [10]

$$
\phi(\vec{\rho}, z)=\frac{1}{L(z)} \Phi(\vec{\xi}, \zeta) \exp \left(i \zeta+i \frac{\dot{L}}{L(z)} \frac{\rho^{2}}{4}\right),
$$

where $\rho=|\vec{\rho}|, L(z)$ is the beam width, and independent variables are defined as

$$
\vec{\xi}=\frac{\vec{\rho}}{L(z)}, \quad \dot{\zeta}=\frac{1}{L^{2}(z)},
$$

we obtain from Eq. (14) the equation for the shape function, $\Phi(\vec{\xi}, \zeta)$, in the form

$$
i \Phi_{\zeta}+\nabla_{\vec{\xi}}^{2} \Phi+|\Phi|^{2} \Phi-\Phi-L^{2} U(\vec{\xi}) \Phi=0
$$

where

$$
U(\vec{\xi})=-\frac{1}{4} \xi^{2} \beta(z) / L^{2}+\epsilon F(\vec{\xi}, L, \vec{R}),
$$

and

$$
\epsilon F(\vec{\xi}, L, \vec{R})=\frac{1}{2} L \ddot{\vec{R}} \vec{\xi}-V(L \vec{\xi}+\vec{R})
$$


with

$$
\ddot{L} L^{3}=-\beta(z) \text {. }
$$

$U(\vec{\xi})$ represents the influence of inertial forces (the centrifugal potential $-(1 / 4) \beta(z) \xi^{2} / L^{2}$ and the potential $(1 / 2) L \ddot{\vec{R}} \vec{\xi}$ of accelerated centroid motion) and of the potential $[-V(L \vec{\xi}+\vec{R})]$, not found in the homogeneous case, on the beam dynamics. When $L(z)$ is known, Eqs. (15), (19) and (20) describe the beam evolution.

Let us consider the beam evolution in the presence of the linear potential $V(\vec{r})$. Basically, one may distinguish two different types of inhomogeneities: broad inhomogeneities and narrow ones. In the case of broad inhomogeneities, i.e., when

$$
L\left|\frac{\nabla_{\vec{r}} V(\vec{r})}{V(\vec{r})}\right| \ll 1,
$$

the inhomogeneity potential $V(\vec{r})$ is a smooth function and its Taylor expansion may be used. The case of parabolic potential $V(\vec{r})$ was studied in Refs. [2,3]. It was shown that the attractive parabolic potential stabilizes subcritical beams $\left(N<N_{c}\right)$ but facilitates the collapse of super-critical ( $N$ $>N_{c}$ ) beams. In the case when the characteristic length scale of inhomogeneity is comparable with the width of the beam

$$
L\left|\frac{\nabla_{\vec{r}} V(\vec{r})}{V(\vec{r})}\right| \geqslant 1,
$$

the polynomial approximation is no longer valid. As in our previous paper [6], we assume that the inhomogeneity potential (7) is weak $(\epsilon \ll 1)$ and super criticality is small: the mass of the beam only slightly exceeds the critical value, i.e., $(N$ $\left.-N_{c}\right) / N_{c} \ll 1$.

The function $\phi(\vec{\rho}, z)$ which describes the beam dynamics in the noninertial frame of reference may be represented as

$$
\phi(\vec{\rho}, z)= \begin{cases}\phi_{s} & \text { if }|\vec{\rho}| \leqslant \xi_{s} L(z), \\ \phi_{o} & \text { if }|\vec{\rho}|>\xi_{s} L(z),\end{cases}
$$

where $\phi_{s}$ is the inner core function, $\phi_{o}$ is its outer part. $\xi_{s}$ $\gg 1$ is a constant that characterizes the size of the beam. The mass of the inner core of the beam (in what follows we will call this part of the beam mass "the core mass") is

$$
N_{s}=\int_{|\vec{\rho}| \leqslant \xi_{s} L(z)}|\phi(\vec{r}, z)|^{2} d \vec{\rho}=\int_{|\vec{\xi}| \leqslant \xi_{s}}|\Phi(\vec{\xi}, \zeta)|^{2} d \vec{\xi} .
$$

Using the solvability condition for the asymptotic expansion of the self-similar shape function $\Phi(\vec{\xi}, \zeta)([11-16,6])$ we obtain the following equations for the centroid $\vec{R}(z)$ and the width $L(z)$ motion:

$$
\frac{1}{2} N_{c} \ddot{\vec{R}}-\nabla_{\vec{R}} \mathcal{V}(L, \vec{R})=0
$$

and

$$
\ddot{L}=-\frac{v}{L^{3}}+\frac{1}{2 M} \frac{\partial}{\partial L} \mathcal{V}(L, \vec{R}) .
$$

Here,

$$
v=\frac{N_{s}-N_{c}}{M}
$$

is the excess core mass above the critical,

$$
\mathcal{V}(L, \vec{R})=\frac{1}{L^{2}} \int_{-\infty}^{\infty} V(\vec{r}) \Psi^{2}\left(\frac{|\vec{r}-\vec{R}|}{L}\right) d x d y
$$

is an effective potential caused by the presence of the linear potential $V(\vec{r})$ where $\Psi(\xi)$ is the so-called ground state or Townes soliton that is the nodeless solution of the equation

$$
\begin{gathered}
\frac{d^{2} \Psi(\xi)}{d \xi^{2}}+\frac{1}{\xi} \frac{d \Psi(\xi)}{d \xi}+\Psi^{3}(\xi)-\Psi(\xi)=0, \\
\left.\frac{d \Psi(\xi)}{d \xi}\right|_{\xi=0}=0 \\
\Psi(\xi) \rightarrow 0 \text { for } \xi \rightarrow \infty .
\end{gathered}
$$

It is worth noting that the Townes soliton is the radially symmetric solution to Eq. (3).

The Townes soliton has a critical mass

$$
2 \pi \int_{0}^{\infty} \Psi^{2}(\xi) \xi d \xi=N_{c} \simeq 11.7,
$$

and in the homogeneous case $[V(\vec{r})=0]$ its Hamiltonian $H$ $=0$. The quantity

$$
M=\frac{1}{4} \int|\vec{\xi}|^{2} \Psi^{2} d \vec{\xi} \approx 3.4
$$

is the second moment of the Townes soliton. Equation (26) coincides with Eq. (15) if $\psi(\vec{r}, z)$ in this equation is the Townes soliton.

Equations (26) and (27) describe the beam dynamics (subcritical beams for beams for $v<0$ and super-critical beams for $v>0$ ) in the adiabatic approximation when the mass of the beam is assumed to be constant $(\dot{v}=0)$ [18]. This approximation is too crude, however, and is not sufficient in the case under consideration because as it is seen from the results of numerical simulations, the beam evolution is accompanied by a radiation.

We shall obtain an equation for $N_{s}(z)$ by considering the radiation rate for the core mass. For this purpose, it is convenient to rewrite Eqs. (19) and (20) as the Schrödinger equation

$$
i \Phi_{\zeta}=-\nabla_{\xi}^{2} \Phi+\mathcal{U}(\vec{\xi}) \Phi
$$




$$
\mathcal{U}(\vec{\xi})=1-\frac{1}{4} \beta \xi^{2}+\frac{1}{2} L^{3} \ddot{\vec{R}} \vec{\xi}-L^{2} V(L \vec{\xi}+\vec{R})-|\Phi|^{2} .
$$

The shape of the potential $\mathcal{U}(\vec{\xi})$ for the case when the inhomogeneity potential $V(\vec{r})$ is a rectangular potential well is given by Eq. (7). The potential energy of inertial forces $\left[(1 / 4) \beta \xi^{2}\right.$ and $\left.(1 / 2) L^{3} \ddot{\vec{R}} \vec{\xi}\right]$ makes the function $U(\xi)$ unbounded from below, and as a result, the motion of a particle in this potential becomes infinite. We are interested in the solutions of Eq. (33) under the boundary condition that the waves are outgoing at $\xi \rightarrow \infty$. With this boundary condition, the problem (33) is no longer self adjoint (see a very lucid discussion of this subject for a closely related problem in [17]). The eigenvalues may have a finite imaginary part that gives the rate of radiation losses.

In the case of the immobile beam placed in the center of the well (7), the potential $\mathcal{U}$ is radially symmetric and outgoing radiation waves are also radially symmetric. However, the accelerated center of motion potential $\left[(1 / 2) L^{3} \ddot{\vec{R}} \vec{\xi}\right]$ significantly modifies the potential profile $\mathcal{U}(\vec{\xi})$, making the profile asymmetric and facilitating the escape in the direction of the inertial force $\ddot{\vec{R}}$.

The evolution of the core mass $N_{s}(z)$ is governed by the equation

$$
\dot{N}_{s}=-\frac{\gamma}{L^{2}} N_{s}
$$

or in terms of the excess mass (28)

$$
\dot{v}=-\frac{\gamma}{L^{2}}\left(\frac{N_{c}}{M}+v\right)
$$

The derivation of the expression for the radiation rate $\gamma$ is rather cumbersome and is given in Appendix B. Here we present only the final result. The raditation rate $\gamma$ may be represented as the sum

$$
\gamma=\theta(\beta) \gamma_{+}+\theta(-\beta) \gamma_{-},
$$

where the radition rates for positive centrifugal coefficient $\left(\gamma_{+}\right)$and negative centrifugal coefficient $\left(\gamma_{-}\right)$have the form

$$
\begin{aligned}
\gamma_{+}= & \frac{\beta^{1 / 4}}{4} \sqrt{\frac{\pi\left(q^{2}+1\right)}{2 q}} \operatorname{erf}\left(\mu \beta^{1 / 4} \frac{\left(q^{2}+1\right)}{\sqrt{2 q}}\right) \\
& \times \exp \left\{-\frac{2}{\sqrt{\beta}}\left[\left(q^{2}+1\right) \arccos \left(\frac{q}{\sqrt{q^{2}+1}}\right)-q\right]\right\} \\
& +\frac{\sqrt{\beta}}{2\left(q^{2}+1\right)(\mu+\sinh \mu)} \\
& \times\left[\exp \left\{-\pi \frac{q^{2}+1}{\sqrt{\beta}}\right\}\right.
\end{aligned}
$$

$$
\begin{aligned}
& -\exp \left\{-2 \frac{q^{2}+1}{\sqrt{\beta}}\left[\frac{\pi}{2}+\left(1+\frac{\mu}{\sinh (\mu)}\right)\right.\right. \\
& \left.\left.\left.\times \arcsin \left(\frac{q}{\sqrt{q^{2}+1}}\right)\right]\right\}\right], \\
\gamma_{-}= & \theta\left(q^{2}-1\right) \frac{|\beta|^{1 / 4}}{4} \sqrt{\frac{\pi q^{3}}{2\left(q^{2}-1\right)}} \operatorname{erf}\left(\pi \sqrt{\frac{q^{2}-1}{2 q \sqrt{|\beta|}}}\right) \\
& \times \exp \left[-\frac{2}{\sqrt{|\beta|}}\left(q-\left(q^{2}-1\right) \operatorname{arcsinh}\left[\frac{1}{\sqrt{q^{2}-1}}\right]\right)\right],
\end{aligned}
$$

and

$$
\gamma=\frac{\sqrt{\pi L^{3}|\ddot{\vec{R}}|}}{2} \exp \left\{-\frac{8}{3 L|\ddot{R}|}\right\} \quad \text { when } \beta=0 .
$$

Here, $\operatorname{erf}(x)$ is the error function $[19,20]$ and the notations

$$
q=\frac{|\ddot{\vec{R}}| L^{3}}{2 \sqrt{|\beta|}}, \quad \mu=\frac{2 \sqrt{3} q}{\sqrt{2 q^{2}+3}}
$$

are used.

The set of Eqs. (26), (27), and (35) together with the expressions

$$
\begin{gathered}
\beta=v-\frac{L^{3}}{2 M} \frac{\partial \mathcal{V}}{\partial L}, \\
q=\frac{L^{3}}{N_{c} \sqrt{|\beta|}}\left|\nabla_{\vec{R}} \mathcal{V}\right|,
\end{gathered}
$$

for the centrifugal and inertial coefficients describes the beam dynamics beyond the adiabatic approximation. The exact analytic expression for the Townes soliton is unknown. Therefore, we use its Gaussian approximation in the form

$$
\Psi_{g}(\xi)=\sqrt{\frac{N_{c}}{\pi B^{2}}} \exp \left\{-\frac{\xi^{2}}{2 B^{2}}\right\},
$$

where $B^{2}=0.8$. Inserting Eq. (42) into Eq. (29), we obtain that in the case of the rectangular well inhomogeneity potential $V(\vec{r})$ given by Eq. (7) and the Townes soliton given by Eq. (42), the effective potential has the form

$$
\mathcal{V}(L, \vec{R})=\frac{5 N_{c}}{2 L^{2}} \int_{0}^{a} r I_{0}\left(\frac{5 r R}{2 L^{2}}\right) \exp \left\{-\frac{5}{4} \frac{r^{2}+R^{2}}{L^{2}}\right\} d r,
$$

where $R=|\vec{R}|$ and $I_{n}(x)$ is the modified Bessel function [19]. We solve numerically the set of Eqs. (26), (27), and (35) which for the effective potential $\mathcal{V}(L, \vec{R})$ given by Eq. (43) take the form 


$$
\begin{gathered}
\ddot{\vec{R}}=-\frac{5 a \epsilon}{L^{2}} I_{1}\left(\frac{5 a R}{2 L^{2}}\right) \exp \left\{-\frac{5}{4} \frac{a^{2}+R^{2}}{L^{2}}\right\} \frac{\vec{R}}{|\vec{R}|}, \\
\ddot{L}=-\frac{v}{L^{3}}-\frac{9 a \epsilon}{2 L^{3}}\left[a I_{0}\left(\frac{5 a R}{2 L^{2}}\right)-R I_{1}\left(\frac{5 a R}{2 L^{2}}\right)\right] \\
\quad \times \exp \left\{-\frac{5}{4} \frac{a^{2}+R^{2}}{L^{2}}\right\}, \\
\dot{v}=-\frac{\gamma}{L^{2}}(3.4+v) .
\end{gathered}
$$

The parameters used are

$$
\begin{gathered}
v(0)=0.01, \quad \epsilon=0.1, \quad a=1, \\
L(0)=2, \quad \dot{L}(0)=0 .
\end{gathered}
$$

We study both types of centroid motion: rectilinear motion and orbital motion.

Rectilinear motion. We investigate the beam dynamics for the following four initial conditions:

$$
\begin{gathered}
\dot{X}(0)=0, \quad X(0)=1,2.5,4,5, \\
\dot{Y}(0)=0, \quad Y(0)=0 .
\end{gathered}
$$

The results of the simulations are presented in Figs. 8-11. As is seen for a given degree of super-criticality $v$ and strength of the inhomogeneity $\epsilon$, the beam evolution depends on the initial distance between the beam and the center of the inhomogeneity potential. Beam collapses when its centroid is either too close to the inhomogeneity $[X(0)=1]$ or too far away from it $[X(0)=5]$. Collapse arresting and stabilizing of the excitation takes place for $X(0)=2.5$ while for $X(0)$ $=4$ the excitation disperses. These results are in qualitative agreement with numerical studies presented in the previous section. It is also worth noting their close relation to the results obtained in Ref. [6] for the one-dimensional quintic nonlinear Schrödinger equation.

Orbital motion. Being interested in the case when the beam is orbiting around the center of inhomogeneity, it is convenient to introduce the polar centroid variables: $\vec{R}$ $=R(\cos \chi, \sin \chi)$. In terms of these variables, Eq. (44) takes the form

$$
\begin{gathered}
\ddot{R}=\frac{m^{2}}{R^{3}}-\frac{5 a \epsilon}{L^{2}} I_{1}\left(\frac{5 a|\vec{R}|}{2 L^{2}}\right) \exp \left\{-\frac{5}{4} \frac{a^{2}+|\vec{R}|^{2}}{L^{2}}\right\}, \\
R^{2} \dot{\chi}=m,
\end{gathered}
$$

where the conserved quantity $m$ is the orbital momentum. In our simulations of Eqs. (45), (46), and (48) we used $m$ $=0.1$ and the following initial conditions

$$
\dot{R}(0)=0, \quad R(0)=2, \quad 3, \quad 5 .
$$

B
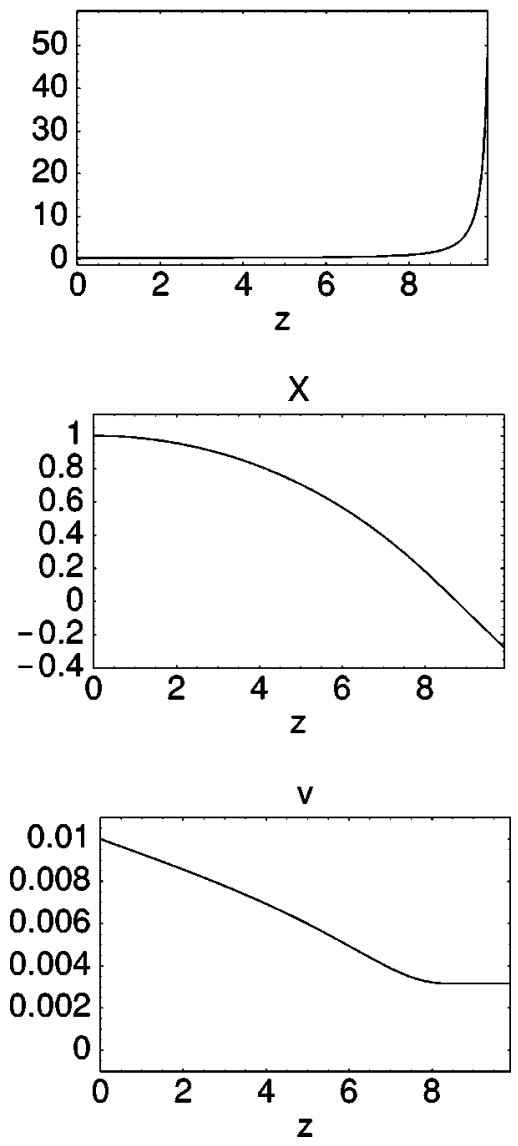

FIG. 8. The half diameter $a$ and the height $\epsilon$ of the potential are given by 2 and 0.1 , respectively. The following initial conditions for $X, \dot{X}, Y, \dot{Y}, L, \dot{L}$, and $v$ are used: $X(0)=1, \dot{X}(0)=0, Y(0)$ $=0, \dot{Y}(0)=0, L(0)=2, \dot{L}(0)=0$, and $v(0)=0.01$. In the upper figure, the inverse width squared $B=1 / L^{2}$ is shown as function of $z$ in dimensionless units. In the middle figure, the $x$ component $X$ of the centroid is depicted, and finally, the lower figure shows the $z$ dependence of $v$ in dimensionless units.

The results of our calculations are presented in Figs. 12-14. Like in the case of the rectilinear motion, the beam collapses when it is either too close or too far away from the center of the inhomogeneity. But it survives when the radiation effects are strong enough to get rid of an excess mass. In our case, it happens when the center of beam is initially at $R(0)=3$.

It is worth stressing that the role of inertial forces in the tunneling effects here is crucial: in the vicinity of inhomogeneity, the inertial forces $(\ddot{\vec{R}}$ and $\beta$ ) are significant, the radiation rate increases, and therefore the mass of the beam decreases with $z\left(N_{s}=N_{c}+M v\right)$. Our results show also that the centroid motion and variations of the width of the beam and its mass are obviously correlated.

\section{SUMMARY}

In this paper, we have studied the interaction of excitations and potentials in the framework of the 2D cubic nonlinear Scrödinger equation. In particular, it has been shown how the critical self focusing of an excitation (beam) whose 
B
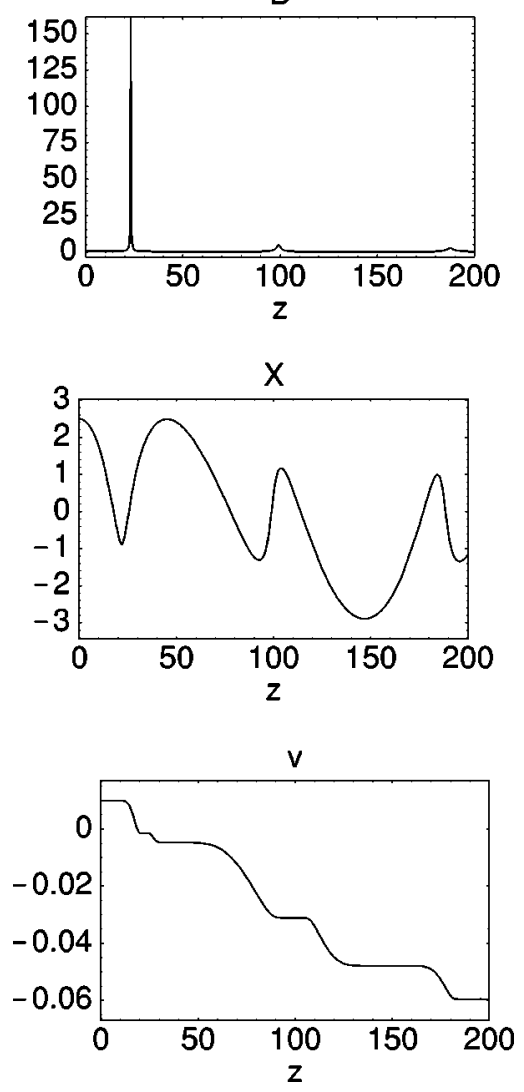

FIG. 9. Same as Fig. 8 with $X(0)=2.5$.

mass is above the threshold for collapse, may be arrested when propagating in the vicinity of a narrow attractive potential. This phenomenon is clearly evident from a series of numerical experiments, divided into two groups characterizing the type of beam motion in the transverse plane perpendicular to the direction of propagation; rectilinear motion where the beam center is limited to motion on a straight line through the center of the potential and orbital motion where the beam center moves in circlelike orbits. For both types of beam motion, numerical calculations showed that oscillations in beam amplitude, width, and center follow the arrest of critical selffocusing. The origin of the observed phenomenon is ascribed to the acceleration of the beam center induced by the potential, either the magnitude of the center (rectilinear motion) or the direction (orbital motion). We employed methods from linear quantum mechanics to understand the collapse arresting. Using two transformations, first, a moving frame of reference centered at the beam center is introduced and second, the lens transformation is used, enables us to calculate the radiation of "mass" from the beam. In qualitative agreement with the numerically obtained results, the analytical appoach establishes a relation between the acceleration of the beam center and the collapse dynamics. Indeed, it is shown how "mass radiation" may bring the beam mass below the threshold for collapse when the beam is subject to strong acceleration in the potential. However, whereas the analytical approach explains the observed phenomenon in clear intuitive terms, it is not suited for quantitative studies of how the beam propagation depends on vari-
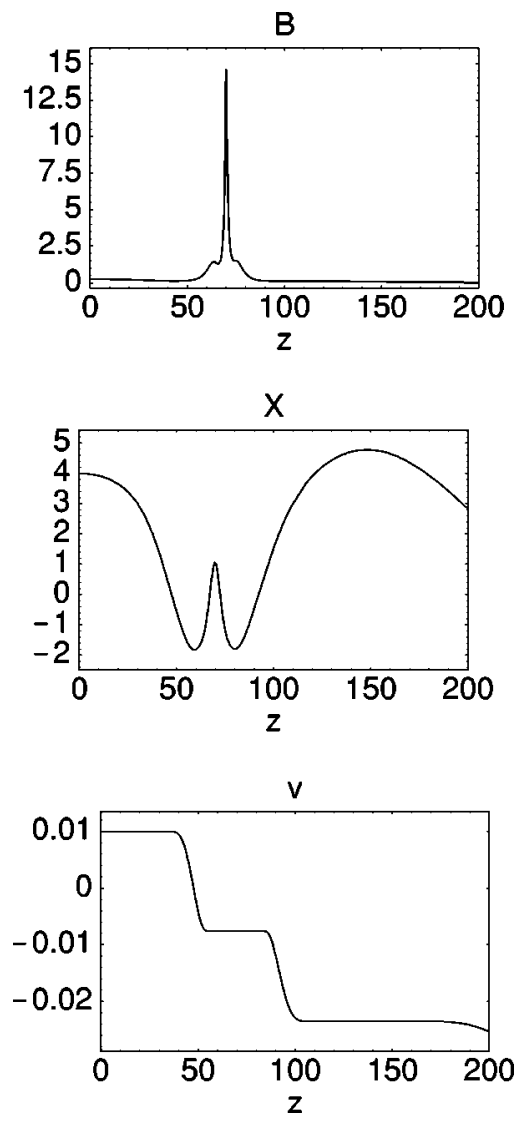

FIG. 10. Same as Fig. 8 with $X(0)=4$.

ous initial parameters. To meet this end, one must resolve to numerical calculations. Two main reasons for the lack of quantitative agreement may be underlined: first, at leading order, it is assumed that the solution core is almost self similar, close to the Townes mode, i.e., the explicit variations of the core versus $z$ are disregarded. Second, the Townes mode is approached by a Gaussian ansatz. These two approximations may contribute to discrepancies between numerics and analytical predictions.

\section{ACKNOWLEDGMENTS}

J. B. Keller and Jens Juul Rasmussen are thanked for helpful discussions. Yu.B.G. thanks MIDIT and the Department of Mathematical Modelling, Technical University of Denmark for hospitality.

\section{APPENDIX A: NUMERICAL METHOD}

To accurately determine the collapse disstance $z_{0}$ we must use a numerical scheme that is capable of resolving the steep gradients and high amplitudes that arise in the beam profile when $z$ approaches $z_{0}$. To meet this end, we integrate the homogeneous version of Eq. (1),

$$
i \frac{\partial \psi}{\partial z}+\frac{\partial^{2} \psi}{\partial x^{2}}+\frac{\partial^{2} \psi}{\partial y^{2}}+|\psi|^{2} \psi=0,
$$



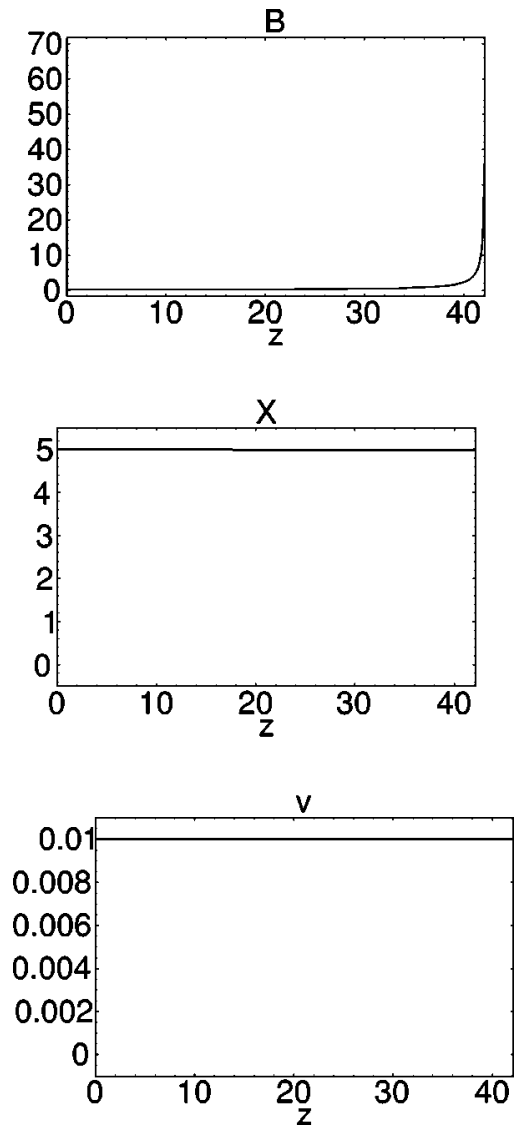

FIG. 11. Same as Fig. 8 with $X(0)=5$.

using a Crank-Nicholson scheme with adaptive integration step on a nonuniform grid.

In a nonuniform grid, the distance between adjacent gridpoints varies across the grid. The grid used here is built as a structure composed of three zones; the inner propagation zone, the outer propagation zone, and the radiation zone. The inner propagation zone $M_{1}$ is characterized by $r \in\left[0, R_{1}[\right.$, the outer propagation zone, $M_{2}$, by $r \in\left[R_{1}, R_{2}\right]$, and the radiation zone $M$ by $\left.r \in] R_{2}, R\right]$, where $R_{1}<R_{2}<R$. The distance between neighboring gridpoints in $M_{1}$ is denoted $\rho_{1}$ and is constant across $M_{1}$. This is also true in the radiation zone $M$ with $\rho_{1}$ replaced by $\rho$. In the outer propagation zone $M_{2}$, which connects $M_{1}$ and $M$, the intergridpoint distance changes smoothly from $\rho_{1}$ at $r=R_{1}$ to $\rho$ at $r=R_{2}$. This way of constructing the nonuniform grid is contrary to the approach where the distance between adjacent gridpoints is allowed to change discontinously across one or more locations in the grid.

When numerically solving Eq. (A1) on the nonuniform grid, we transform the non-equidistantly positioned $r$ gridpoints into a uniform grid, denoted the $u$ grid. If the relation between $r$ and $u$ is given by

$$
\begin{aligned}
& u=F(r), \\
& r=G(u),
\end{aligned}
$$

then
B

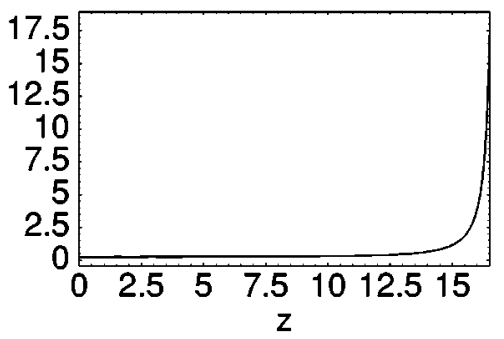

R
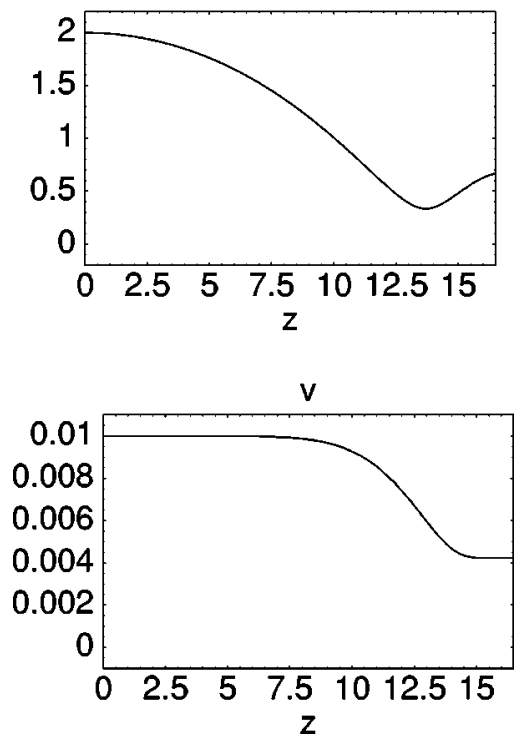

FIG. 12. The half diameter $a$ and the height $\epsilon$ of the potential are given by 2 and 0.1 , respectively. The following initial conditions for $R, \dot{R}, L, \dot{L}$, and $v$ are used: $R(0)=2, \dot{R}(0)=0, L(0)$ $=2, \dot{L}(0)=0$, and $v(0)=0.01$. In the upper figure, the inverse width squared $B=1 / L^{2}$ is shown as a function of $z$ in dimensionless units. In the middle figure, the magnitude $R$ of the centroid is depicted, and finally, the lower figure shows the $z$ dependence of $v$ in dimensionless units.

$$
\phi(u, z)=\psi(G(u), z) .
$$

Insertion of Eqs. (A2) and (A3) into Eq. (A1) yields

$$
\begin{aligned}
& i \frac{\partial \phi}{\partial z}+\left(\frac{1}{G(u) G^{\prime}(u)}-\frac{G^{\prime \prime}(u)}{G^{\prime}(u)^{3}}\right) \frac{\partial \phi}{\partial u}+\frac{1}{G^{\prime}(u)^{2}} \frac{\partial^{2} \phi}{\partial u^{2}}+|\phi|^{2} \phi \\
& =0 .
\end{aligned}
$$

Solving the standard cubic NLSE on the nonuniform $r$ grid is thus equivalent to solving an NLSE with $u$-dependent coefficients on the uniform $u$ grid. For the transformation function, $G(u)$, we use

$$
G(u)=\alpha u, u \in\left[0, U_{1}\right],
$$

$$
\begin{aligned}
G(u)= & R_{1}+\alpha\left(u-U_{1}\right)+a\left(\frac{\left(u-U_{1}\right)^{6}}{30}-\frac{\Delta_{U}\left(u-U_{1}\right)^{5}}{10}\right. \\
& \left.+\frac{\Delta_{U}^{2}\left(u-U_{1}\right)^{4}}{12}\right)
\end{aligned}
$$


B

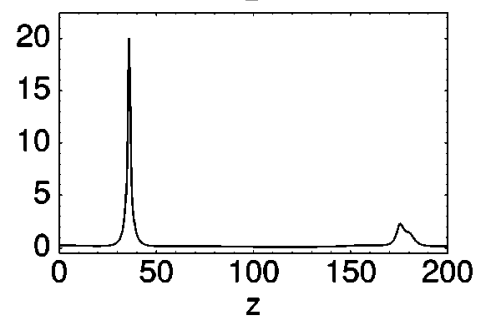

$\mathrm{R}$

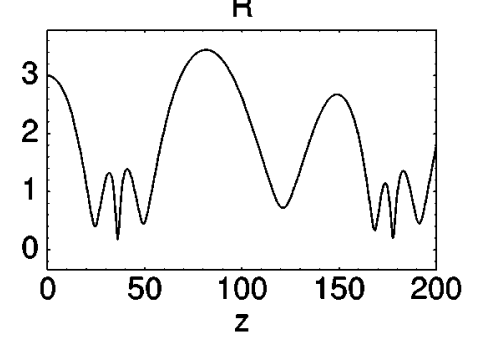

v

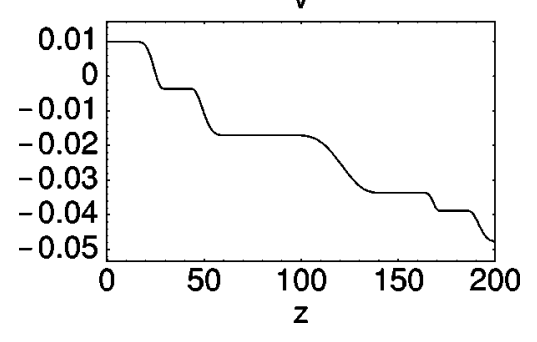

FIG. 13. Same as Fig. 12 with $R(0)=3$.

$$
\begin{gathered}
u \in\left[U_{1}, U_{2}\right], \\
G(u)=R_{2}+\beta\left(u-U_{2}\right), u \in\left[U_{2}, U\right],
\end{gathered}
$$

where

$$
\begin{gathered}
U_{1}=\frac{R_{1}}{\alpha}, \\
U_{2}=U_{1}+\Delta_{U}, \\
U=U_{2}+\frac{\left(R_{2}-R_{1}\right)}{\beta}, \\
a=\frac{30(\beta-\alpha)}{\Delta_{U}^{5}}, \\
\Delta_{U}=\frac{2\left(R_{2}-R_{1}\right)}{\alpha+\beta}, \\
\frac{\alpha}{\beta}=\frac{\rho_{1}}{\rho} .
\end{gathered}
$$

From the above relations, it is evident how the structure of the grid is uniquely determined from $R_{1}, R_{2}, R, \rho_{1}$, and $\rho$, once either $\alpha$ or $\beta$ has been chosen as an arbitrary positive number.
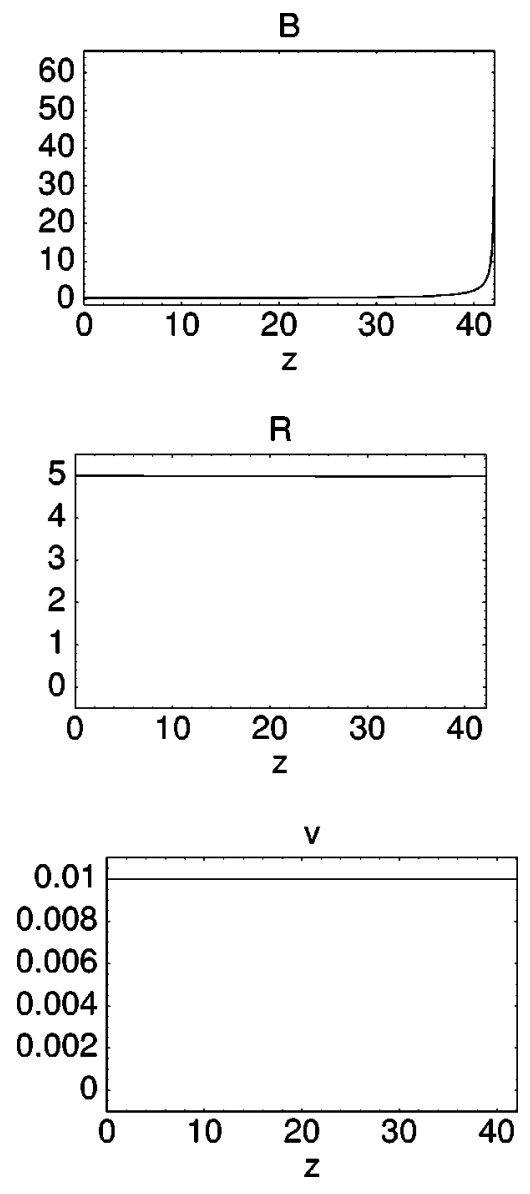

FIG. 14. Same as Fig. 12 with $R(0)=5$.

As self-focusing sets in, the part of the beam that lies in the outer propagation zone at $z=0$ gradually moves towards the inner propagation zone with the high density of gridpoints. Radiation, which in the process of self focusing is separated from the high-intensity part of the beam (the core), is allowed to freely disperse in the outer propagation zone and the radiation zone. Once the major part of the beam mass is located in $M_{1}$, the beam width has decreased significantly and the Crank-Nicholson integration scheme requires a small integration step due to the high amplitudes and steep gradients. To meet this end, the integration step $\Delta_{z}$ is allowed to adapt to the shape of the envelope function. Let $|\psi|_{\text {max }}$ at $z$ $=z_{1}$ be defined as

$$
|\psi|_{\max }=\max \left\{|\psi|, r \in[0, \mathrm{R}], z=z_{1}\right\} .
$$

To first order in the integration step $\Delta_{z}, \psi$ changes from $z$ $=z_{1}$ to $z=z_{1}+\Delta_{z}$, according to

$$
\Delta \psi=\left.i\left(\frac{1}{r} \frac{\partial \psi}{\partial r}+\frac{\partial^{2} \psi}{\partial r^{2}}+|\psi|^{2} \psi\right)\right|_{z=z_{1}} \Delta_{z} .
$$

$\Delta \psi$ is everywhere in the $r$ domain required to obey the relation 


$$
\frac{|\Delta \psi|}{|\psi| \max }<\gamma
$$

where $\gamma$ is a small parameter specifying the allowed change in $\psi$. From Eq. (A7) and Eq. (A8), an upper bound for $\Delta_{z}$ is found

$$
\Delta_{z}=\min \left\{\frac{\gamma|\psi|_{\max }}{\left.\left.\left|\frac{1}{r} \frac{\partial \psi}{\partial r}+\frac{\partial^{2} \psi}{\partial r^{2}}+\right| \psi\right|^{2} \psi\right|_{z=z_{1}}}, r \in[0, \mathrm{R}]\right\}
$$

In order to limit the possibility for zero in the denominator, the inequality Eq. (A10) is strengthened by requiring

$$
\Delta_{z}=\min \left\{\frac{\gamma|\psi|_{\max }}{\left(\left|\frac{1}{r} \frac{\partial \psi}{\partial r}+\frac{\partial^{2} \psi}{\partial r^{2}}\right|+|\psi|^{3}||_{z=z_{1}}\right.}, r \in[0, \mathrm{R}]\right\}
$$

On the uniform $u$ grid, Eq. (A11) reads

$$
\Delta_{z}=\min \left\{\frac{\gamma \max \left\{|\phi|, u \in[0, \mathrm{U}], z=z_{1}\right\}}{\left.||-\frac{G^{\prime \prime}(u)}{G^{\prime}(u)^{3}} \frac{\partial \phi}{\partial u}+\left.\frac{1}{G^{\prime}(u)^{2}} \frac{\partial^{2} \phi}{\partial u^{2}}|+| \phi\right|^{3}\right)\left.\right|_{z=z_{1}}}, u \in[0, \mathrm{U}]\right\}
$$

Next, we apply the algoritm to a beam with initial conditions given by

$$
\psi(x, y, z=0)=A e^{-\left(x^{2}+y^{2} / 2 w^{2}\right)},
$$

where $A=2$ and $w=0.975$. The NLSE, Eq. (A1), is solved on a grid characterized by $R_{1}=0.02, R_{2}=2.09, R=20.87$, $\alpha / \beta=1 / 160$, and $\gamma=0.025$. The Crank-Nicholson solver is iterated until $z=5.45$ where the amplitude at $r=0$ is 159.7 , an eighty times increase of the initial amplitude. The nonuniform grid is not capable of resolving the steep gradients in $\psi$ beyond this propagation distance, and we thus approximate the collapse distance $z_{0}$ with the propagation distance $z$ $=5.45$.

\section{APPENDIX B: RADIATION RATE}

In this appendix, we derive an equation for the radiation rate for the core mass. We look for the solution of Eq. (33) in the form

$$
\Phi(\vec{\xi}, \zeta)=\chi(\vec{\xi}) e^{i \delta \zeta},
$$

where the eigenvalue $\delta$ and the shape function $\chi$ are determined from the equation

$$
\begin{gathered}
-\nabla_{\xi}^{2} \chi+\mathcal{U}(\vec{\xi}) \chi=\lambda \chi, \\
\mathcal{U}(\vec{\xi})=\mathcal{U}_{c}(\vec{\xi})+\mathcal{U}_{f}(\vec{\xi}), \\
\mathcal{U}_{c}(\vec{\xi})=-|\Phi|^{2}, \\
\mathcal{U}_{f}(\vec{\xi})=-\frac{1}{4} \beta \xi^{2}+\vec{f} \vec{\xi},
\end{gathered}
$$

where $\lambda=-1-\delta, \vec{f}=(1 / 2) \ddot{\vec{R}} L^{3}$ is the inertial force. It is seen that the potential $\mathcal{U}(\vec{\xi})$ consists of parts with strongly different interaction ranges: $\mathcal{U}_{c}(\vec{\xi})$ is a short-range radially symmetric potential of radius $\xi_{g}$ (which is the radius of the ground-state Townes soliton) and $\mathcal{U}_{f}(\vec{\xi})$ describes the action of the centrifugal force $\left[(1 / 4) \beta \xi^{2}\right]$, inertial force $(\vec{f} \vec{\xi})$. We omitted the inhomogeneity potential $\left[L^{2} V(L \vec{\xi}+\vec{R})\right]$ in the potential $\mathcal{U}(\vec{\xi})$ because due to its weakness and narrow character [see Eq. (7)] it does not change significantly the height and width of the potential profile $\mathcal{U}(\vec{\xi})$. We are looking for solutions of the eigenvalue problems (B2)-(B4) under outgoing wave boundary condition. In this case, this problem is not self adjoint and the eigenvalues $\lambda$ may be complex. It was shown in [21] (see also [22]) that in the limit of small $\xi_{g}$, the equation for eigenvalues has the form

$$
\begin{aligned}
& \lim _{\vec{\xi} \rightarrow 0} \lim _{\eta \rightarrow+0} \int_{0}^{\infty}\left[G_{0}(\vec{\xi}, t \mid 0,0) e^{i \lambda_{0} t}-G_{f}(\vec{\xi}, t \mid 0,0) e^{i \lambda t}\right] e^{-\eta t} d t \\
& \quad=0
\end{aligned}
$$

where $\lambda_{0}$ is an unperturbed eigenvalue in the short-range potential (B3). In our case, $\lambda_{0}=-1$ is the eigenfrequency of the ground-state Townes soliton. $G_{0}\left(\vec{\xi}, t \mid \vec{\xi}^{\prime}, t^{\prime}\right)$ is the Green function of free motion. It satisfies the equation

$$
\left(i \frac{\partial}{\partial t}+\nabla_{\vec{\xi}}^{2}\right) G_{0}\left(\vec{\xi}, t \mid \vec{\xi}^{\prime}, t^{\prime}\right)=i \delta\left(t-t^{\prime}\right) \delta\left(\vec{\xi}-\vec{\xi}^{\prime}\right),
$$

and in the two-dimensional case has the form [23]

$$
G_{0}\left(\vec{\xi}, t \mid \vec{\xi}^{\prime}, t^{\prime}\right)=-\frac{1}{2 \pi\left(t-t^{\prime}\right)} \exp \left\{i \frac{\left(\vec{\xi}-\vec{\xi}^{\prime}\right)^{2}}{2\left(t-t^{\prime}\right)}\right\} .
$$


The Green function $G_{f}\left(\vec{\xi}, t \mid \vec{\xi}^{\prime}, t^{\prime}\right)$ satisfies the equation

$$
\left(i \frac{\partial}{\partial t}+\nabla_{\vec{\xi}}^{2}-\mathcal{U}_{f}(\vec{\xi})\right) G_{f}\left(\vec{\xi}, t \mid \vec{\xi}^{\prime}, t^{\prime}\right)=i \delta\left(t-t^{\prime}\right) \delta\left(\vec{\xi}-\vec{\xi}^{\prime}\right)
$$

and describes the motion in the field of inertial forces (B4). The potential (B4) is quadratic. Therefore, the Green function may be calculated explicitly [23] and in the twodimensional case, may be represented as follows:

$$
G_{f}\left(\vec{\xi}, t \mid \vec{\xi}^{\prime}, t^{\prime}\right)=-\frac{\sqrt{-\beta}}{2 \pi \sin \sqrt{-\beta}\left(t-t^{\prime}\right)} e^{i S\left(\vec{\xi}, t \mid \vec{\xi}^{\prime}, t^{\prime}\right)},
$$

where

$$
\begin{aligned}
S\left(\vec{\xi}, t \mid \vec{\xi}^{\prime}, t^{\prime}\right)= & \frac{\sqrt{-\beta}}{2 \sin \left(\sqrt{-\beta}\left(t-t^{\prime}\right)\right)} \\
& \times\left[\left(\vec{\xi}^{2}+\vec{\xi}^{\prime 2}+\frac{2}{\beta} \vec{f}\left(\vec{\xi}+\vec{\xi}^{\prime}\right)+\frac{2}{\beta^{2}} \vec{f}^{2}\right)\right. \\
& \times \cos \left(\sqrt{-\beta}\left(t-t^{\prime}\right)\right) \\
& \left.-2\left(\vec{\xi} \vec{\xi}^{\prime}-\frac{1}{\beta} \vec{f}\left(\vec{\xi}+\vec{\xi}^{\prime}\right)+\frac{\vec{f}^{2}}{\beta^{2}}\right)-\frac{\vec{f}^{2}}{2 \beta}\left(t-t^{\prime}\right)\right]
\end{aligned}
$$

is the classical action

$$
S\left(\vec{\xi}, t \mid \vec{\xi}^{\prime}, t^{\prime}\right)=\int_{t^{\prime}}^{t} L(\tau) d \tau,
$$

where $L=(1 / 4) \dot{\xi}^{2}-\mathcal{U}_{f}$ is the classical Lagrangian function. Inserting Eqs. (B7), (B9), and (B10) into Eq. (B5) we get

$$
\ln \left(\frac{\lambda_{0}}{\lambda}\right)=2 \pi \int_{0}^{\infty} g(t) e^{i \lambda t} d t,
$$

with

$$
\begin{aligned}
g(t) \equiv & G_{f}(0, t \mid 0,0)-G_{0}(0, t \mid 0,0) \\
= & \frac{1}{4 \pi t}\left(1-\frac{\sqrt{-\beta} t}{\sin \sqrt{-\beta}\left(t-t^{\prime}\right)}\right. \\
& \left.\times \exp \left\{-i \frac{2 \vec{f}^{2}}{\beta \sqrt{-\beta}}\left(\frac{\sqrt{-\beta} t}{2}-\tan \frac{\sqrt{-\beta} t}{2}\right)\right\}\right) .
\end{aligned}
$$

The escape rate $\gamma$ is defined as the imaginary part of the eigenvalue $\delta$. In the weak inertial and centrifugal forces limit the escape rate is determined by the expression

$$
\gamma=-2 \pi \operatorname{Im} \int_{0}^{\infty} g(t) e^{-i t} d t .
$$

The escape process for positive and negative $\beta$ is different. One may therefore represent the rate $\gamma$ as the sum

$$
\gamma=\theta(\beta) \gamma_{+}+\theta(-\beta) \gamma_{-},
$$

where $\gamma_{+}\left(\gamma_{-}\right)$is the escape rate in the case of positive(negative) centrifugal force $\beta$. When $\beta>0$ Eq. (B14) takes the form

$$
\begin{gathered}
\gamma_{+}=-\frac{\pi}{4}+I(\nu, \alpha), \\
I(\nu, \alpha)=\operatorname{Im} \int_{0}^{\infty} \frac{\exp \{i \nu(u-\alpha \tanh u)\}}{\sinh (2 u)} d u,
\end{gathered}
$$

where

$$
\nu=2 \frac{f^{2}+\beta}{\beta \sqrt{\beta}}, \quad \alpha=\frac{f^{2}}{f^{2}+\beta} .
$$

When

$$
0<\beta \sim f^{2} \ll 1,
$$

the parameter $\nu \gg 1$. To evaluate the integral (B17), we use the method of steepest descents. To this end, we first deform the original integration path $C$ that runs from 0 to $\infty$ along the real $u$ axis, to the contour in the complex $(u, v)$ plane that consists of six components: $C_{1}$ and $C_{5}$, which are the quarter of circles of the infinitesimally small radius $\epsilon$, surrounding the points $(0,0)$ and $(0, i \pi)$, respectively; $C_{2}$ and $C_{4}$, which run along the imaginary $v$ axis from $i \epsilon$ to $i v_{0}$ and from $i\left(\pi-v_{0}\right)$ to $i(\pi-\epsilon)$, respectively; the contour $C_{3}$, which is determined by the equation

$$
\cos ^{2} v=\alpha \frac{\sinh 2 u}{2 u}-\sinh ^{2} u,
$$

with

$$
0 \leqslant u \leqslant u_{0}, \quad v_{0} \leqslant v \leqslant \pi-v_{0},
$$

where $v_{0}=\arccos \sqrt{\alpha}$ and the parameter $u_{0}$ is determined by the equation $u_{0}$ tanh $u_{0}=\alpha$; and $C_{6}$, which runs parallel to the real $u$ axis from the point $(\epsilon, i \pi)$ to $(\infty, i \pi)$. The contour $C_{3}$ is the steepest-descent path. It was chosen in the form (B20) to have $\operatorname{Im}(z-a \tanh z)=0, \quad z=u+i v$. The integral under consideration may be represented as the sum

$$
\begin{gathered}
I(\nu, \alpha)=\sum_{j=1}^{6} I_{j}(\nu, \alpha), \\
I_{j}(\nu, \alpha)=\operatorname{Im} \int_{C_{j}} \frac{\exp \{i \nu(z-\alpha \tanh z)\}}{\sinh (2 z)} d z .
\end{gathered}
$$

It is easy to see that 


$$
I_{j}(\nu, \alpha)= \begin{cases}\frac{\pi}{4} & \text { if } j=1, \\ 0 & \text { if } j=2 \text { and } 4, \\ \frac{\pi}{4} e^{-\nu \pi} & \text { if } j=5, \\ I(\nu, \alpha) e^{-\nu \pi} & \text { if } j=6 .\end{cases}
$$

Thus, from Eq. (B16), we get

$$
\gamma_{+}=\frac{1}{1-e^{-\nu \pi}}\left(\frac{\pi}{2} e^{-\nu \pi}+I_{3}(\nu, \alpha)\right) .
$$

The integral on the steepest-descent path $I_{3}(\nu, \alpha)$ may be represented as the sum

$$
\begin{aligned}
I_{3}(\nu, \alpha)= & \left(\int_{v_{0}}^{\pi / 2}+\int_{\pi / 2}^{\pi-v_{0}}\right) \\
& \times \frac{\sinh 2 u \cos 2 v-\frac{d u}{d v} \cosh 2 u \sin 2 v}{\sinh ^{2} 2 u+\sinh ^{2} 2 v} e^{-\nu P(v)} d v \\
\equiv & I_{3}^{\prime}(\nu, \alpha)+I_{3}^{\prime \prime}(\nu, \alpha),
\end{aligned}
$$

where

$$
P(v)=v-\alpha \frac{\sin 2 v}{\cos 2 v+\cosh 2 u},
$$

and the dependence $u(v)$ is given by Eq. (B20). The function (B25) is monotonic in both intervals of integration. Therefore, one may evaluate the integrals $I_{3}^{\prime}(\nu, \alpha)$ and $I_{3}^{\prime \prime}(\nu, \alpha)$ for $\nu \gg 1$ by using the Laplace method (see, e.g., [24]). In the leading approximation (neglecting the small terms $e^{-\nu}$ ) we get

$$
\gamma_{+} \approx I_{3}^{\prime}(\nu, \alpha)+I_{3}^{\prime \prime}(\nu, \alpha)
$$

where

$$
\begin{aligned}
I_{3}^{\prime}(\nu, \alpha) \simeq & \frac{1}{4} \pi^{1 / 2} \alpha^{-1 / 4}(1-\alpha)^{-3 / 4} \nu^{-1 / 2} \\
& \times \operatorname{erf}\left(\nu^{1 / 2} \alpha^{-1 / 4}(1-\alpha)^{1 / 4} u_{0}\right) \\
& \times \exp \{-\nu(\arccos \sqrt{\alpha}-\sqrt{\alpha(1-\alpha)})\}, \\
I_{3}^{\prime \prime}(\nu, \alpha) \simeq & \frac{1}{\left(2 u_{0}+\sinh 2 u_{0}\right) \nu} \\
& \times\left(1-\exp \left\{-\nu\left(1+\frac{2 u_{0}}{\sinh 2 u_{0}}\right)\right.\right. \\
& \times \arcsin \sqrt{\alpha}\}) \exp \left\{-\frac{\nu \pi}{2}\right\},
\end{aligned}
$$

where $\operatorname{erf}(x)=(2 / \sqrt{\pi}) \int_{0}^{x} e^{-z^{2}} d z$ is the error function [19].
In the limit of vanishing inertial force $\vec{f}$,

$$
\alpha \rightarrow 0, \quad \nu \rightarrow 2 \beta^{-3 / 2},
$$

and

$$
I_{3}^{\prime}=I_{3}^{\prime \prime} \simeq \frac{1}{2} e^{-(\pi / \sqrt{\beta})} .
$$

Thus, the escaping rate is controlled by the centrifugal force $\beta$ and its form

$$
\gamma_{+} \simeq e^{-(\pi / \sqrt{\beta})}
$$

is the same as in the case of the homogeneous nonlinear Schrödinger equation [13-16]. When the centrifugal force $\beta$ is small

$$
\alpha \rightarrow 1, \quad \nu \rightarrow 2 \vec{f}^{2} \beta^{-3 / 2}
$$

and

$$
I_{3}^{\prime} \simeq \sqrt{\frac{\pi|\vec{f}|}{32}} \exp \left\{-\frac{4}{3|\vec{f}|}\right\}, \quad I_{3}^{\prime \prime} \sim \frac{\beta^{3 / 2}}{|\vec{f}|^{2}} \exp \left\{-\frac{\pi|\vec{f}|^{2}}{\beta^{3 / 2}}\right\}
$$

and the escaping rate (B23) is mainly determined by the inertial force $\vec{f}$

$$
\gamma_{+} \simeq \sqrt{\frac{\pi|\vec{f}|}{32}} \exp \left\{-\frac{4}{3|\vec{f}|}\right\} .
$$

Let us consider now the case of negative centrifugal force $\beta$. It is worth noticing that in contrast to the previous case when the potential $\mathcal{U}(\vec{\xi})$ was unbounded from below, the hermicity of the Hamiltonian (B2) could be violated by using as a boundary condition for $|\xi| \rightarrow \infty$ only outgoing waves, in the case of $\beta<0$, the function $\mathcal{U}(\vec{\xi})$ represents an asymmetric double-well potential. For this case, the concept of the escape rate as an imaginary part of the energy of the particle is obviously not correct. A particle located in the well created by the potential (B3) tunnels to the parabolic well (B4) and will return back to the first well in a finite-time interval. This back-and-forth motion causes the energy shift but not the escape rate.

In the case under consideration, when the parabolic well is almost flat (super criticality is small) and the potential barrier that separates the wells is broad (inertial force $L^{3} \ddot{\vec{R}}$ is weak) one may neglect bouncing of the particle from the opposite side of the parabolic potential well. In this approximation, one may again introduce the notion of the escape rate $\gamma_{-}$but the Eq. (B14) takes now the form 


$$
\begin{aligned}
\gamma_{-}= & -\frac{\pi}{4}-\operatorname{Im} \int_{0}^{T} \frac{\sqrt{-\beta}}{\sin (-\beta) t} \\
& \times \exp \left\{-i \frac{2 \vec{f}^{2}}{\beta \sqrt{-\beta}}\left(\frac{\sqrt{-\beta} t}{2}-\tan \frac{\sqrt{-\beta} t}{2}\right)\right\} d t \\
= & -\frac{\pi}{4}+\operatorname{Im} \int_{0}^{\pi / 2} \frac{e^{-i \nu(u-\alpha \tan u)}}{\sin 2 u} d u,
\end{aligned}
$$

where $T=(\pi / \sqrt{-\beta})$ is the time of the first bounce that is of course the half period of oscillations in the parabolic well and the parameters $\nu$ and $\alpha$ are given by Eq. (B18).

To evaluate the integral in Eq. (B35), we use the method of steepest descents. Here, the original integration path $C$ that runs from 0 to $\pi / 2$ along the real $u$ axis, to the contour in the complex $(u, v)$ plane that consists of six components: $C_{1}$, which is the quarter of circle of the infinitesimally small radius $\epsilon$, surrounding the points $(0,0) ; C_{2}$, which runs along the imaginary $v$ axis from $i \epsilon$ to $i v_{1} \equiv i \operatorname{arccosh} \sqrt{\alpha}$; and the steepest-descent contour $C_{3}$ which is determined by the equation

$$
\sinh ^{2} v=\alpha \frac{\sin 2 u}{2 u}-\cos ^{2} u
$$

Note that here the parameter $\alpha>1$, because only under this condition the bottom of the parabolic well is lower than the energy level in the potential (B3) $\left(\lambda_{0}=-1\right)$ and tunneling to the parabolic well may occur. Proceeding in the same way as in the case of positive $\beta$, we obtain that the escape rate (B35) may be represented as follows:

$$
\begin{aligned}
\gamma_{-}= & \theta(\alpha-1) \frac{1}{4} \sqrt{\frac{\pi}{\nu}}\left(\frac{\alpha}{\alpha-1}\right)^{3 / 4} \\
& \times \operatorname{erf}\left(\frac{\pi}{2} \nu^{1 / 2}\left(\frac{\alpha-1}{\alpha}\right)^{1 / 4}\right) e^{-\nu(\sqrt{(\alpha-1) \alpha}-\operatorname{arcsinh} \sqrt{\alpha-1})} .
\end{aligned}
$$

In the limit, when the cenrifugal force is weak, Eq. (B37) takes the form

$$
\gamma_{-}=\theta(\alpha-1) \sqrt{\frac{\pi|\vec{f}|}{32}} \exp \left\{-\frac{4}{3|\vec{f}|}\right\} \text {, }
$$

which coincides with Eq. (B34) obtained for the case of positive $\beta$.

Combining Eqs. (B15), (B26), (B27), (B28), and (B37), we get

$$
\begin{aligned}
& \gamma=\theta(\beta) \gamma_{+}+\theta(-\beta) \gamma_{-} . \\
& \gamma_{+}=\frac{1}{4} \sqrt{\frac{\pi\left(f^{2}+\beta\right)}{2 f}} \operatorname{erf}\left(\mu \frac{\left(f^{2}+\beta\right)}{\sqrt{2 \beta f}}\right) \exp \left\{-2 \frac{f^{2}+\beta}{\beta \sqrt{\beta}}\left[\frac{\pi}{2}\right.\right. \\
& \left.\left.-\frac{f \sqrt{\beta}}{f^{2}+\beta}-\arcsin \left(\frac{f}{\sqrt{f^{2}+\beta}}\right)\right]\right\} \\
& +\frac{\beta \sqrt{\beta}}{2\left(f^{2}+\beta\right)(\mu+\sinh \mu)}\left(\exp \left\{-\pi \frac{f^{2}+\beta}{\beta \sqrt{\beta}}\right\}\right. \\
& -\exp \left\{-2 \frac{f^{2}+\beta}{\beta \sqrt{\beta}}\left[\frac{\pi}{2}+\left(1+\frac{\mu}{\sinh (\mu)}\right)\right.\right. \\
& \left.\left.\left.\times \arcsin \left(\frac{f}{\sqrt{f^{2}+\beta}}\right)\right]\right\}\right) \text {, } \\
& \gamma_{-}=\theta\left(f^{2}-|\beta|\right) \frac{\sqrt{\pi}}{4} \sqrt{\frac{f^{3}}{2\left(f^{2}-|\beta|\right)}} \operatorname{erf}\left(\pi \sqrt{\frac{f^{2}-|\beta|}{2 f|\beta|}}\right)
\end{aligned}
$$

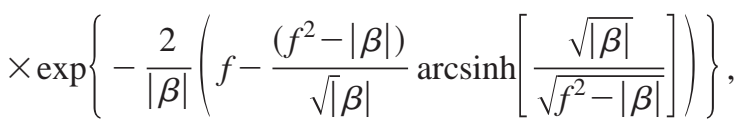

$$
\begin{aligned}
& \frac{\pi}{2}
\end{aligned}
$$

.

where the notation

$$
\mu \equiv \frac{2 \sqrt{3} f}{\sqrt{2 f^{2}+3 \beta}}
$$

is used.
[1] A.C. Newell and J.V. Moloney, Nonlinear Optics (AddisonWesley, Reading, MA, 1992).

[2] L. Bergé, Phys. Plasmas 4, 1227 (1997).

[3] Yu.B. Gaididei, K.Ø. Rasmussen, and P.L. Christiansen, Phys. Rev. E 52, 2951 (1995).

[4] E.V. Shuryak, Phys. Rev. A 54, 3151 (1996).

[5] Y. Kagan, A.E. Muryshev, and G.V. Shlyapnikov, Phys. Rev. Lett. 81, 933 (1998).

[6] Yu.B. Gaididei, J. Schjødt-Eriksen, and P.L. Christiansen, Phys. Rev. E 60, 4877 (1999).

[7] J. Juul Rasmussen and K. Rypdal, Phys. Scr. 33, 481 (1986); K. Rypdal and J. Juul Rasmussen, ibid. 33, 498 (1986).

[8] M.G. Vakhitov and A.A. Kolokolov, Izv. Vyssh. Uchebn.
Zaved Radiofiz. 16, 1020 (1973) [Radiophys. Quantum Electron. 16, 783 (1973)].

[9] H.A. Rose and M.I. Weinstein, Physica D 30, 207 (1988).

[10] V.I. Talanov, Pis'ma Zh. Éksp. Teor. Fiz. 11, 447 (1970) [JETP Lett. 11, 303 (1970)].

[11] G.M. Fraiman, Zh. Eksp. Teor. Fiz. 88, 390 (1985) [Sov. Phys. JETP 61, 228 (1985)].

[12] N.N. Akhmediev, Zh. Éksp. Teor. Fiz. 83, 545 (1982) [Sov. Phys. JETP 56, 299 (1982)].

[13] A.I. Smirnov and G.M. Fraiman, Physica D 52, 2 (1991).

[14] B.J. LeMesurier, G. Papanicolau, C. Sulem, and P. Sulem, Physica D 32, 210 (1988).

[15] M. Landman, G. Papanicolau, C. Sulem, and P. Sulem, Phys. Rev. A 38, 3837 (1988). 
[16] S. Dyachenko, A.C. Newel, A. Pushkarev, and V.E. Zakharov, Physica D 57, 96 (1992).

[17] R.B. Paris and A.D. Wood, IMA J. Appl. Math. 43, 273 (1989).

[18] The same set of equations was obtained in the paper by Yu.B. Gaididei, D. Hendriksen, P.L. Christiansen, and K.Ø. Rasmussen, Phys. Rev. B 58, 3075 (1998) using the collective coordinate approach.

[19] M. Abramowitz and I. Stegun, Handbook of Mathematical Functions (Dover, New York, 1965).

[20] D. Anderson, M. Bonnedal, and M. Lisak, Phys. Fluids 22,
1838 (1979).

[21] S.P. Andreev, B.M. Karnakov, and V.D. Mur, Teor. Mat. Fiz. 64, 287 (1985).

[22] V.S. Popov, B.M. Karnakov, and V.D. Mur, Zh. Éksp. Teor. Fiz. 113, 1579 (1998) [Sov. Phys. JETP 86, 860 (1998)].

[23] R.P. Feynman and A.R. Hibbs, Quantum Mechanics and Path Integrals (McGraw-Hill, New York, 1965).

[24] C.M. Bender and S.A. Orszag, Advanced Mathematical Methods for Scientists and Engineers (McGraw-Hill, New York, 1978). 